\title{
Optimizing Signal Behavior of Femtocells for Improved Network
}

\author{
O. A. Akinlabi and Meera K. Joseph, University of Johannesburg, South Africa
}

\begin{abstract}
The high demand for network coverage in an indoor setting brought about the acceptance of femtocell technology as a solution using the backhaul connectivity in the existing network. The quality of signal, voice calling, Internet, security and data are improved through the use femtocell at the indoor environment. Here the service provider attempts to reduce their operation cost by presenting self-organizing mechanisms for optimization of the network. The remarkable part is that, femtocells improves coverage, enhances the data rate at the indoor environment. Therefore, the challenges of the femtocell also known as interference deteriorates the capacity and quality performance of the whole cellular network. In this paper we simulate the bit error rate against signal behaviour at the indoor environment and we also simulate the transmitting power over signal for both macrocells and femtocells. We focus on the transmitting power that might cause interference within the cellular network.
\end{abstract}

Index Terms - Femtocells, Macrocells, Signal behavior, Transmitting Power.

\section{INTRODUCTION}

$\mathrm{M}$ ost users of mobile network demand a quality performance of service such as voice calling, data, Internet and better signal within an indoor environment. The failure of macrocell in order to achieve the above mentioned has brought about femtocell technology. However, the deployment of femtocell has drawn the attention of researchers, academics and experts in telecommunication industry over macrocell for improved coverage and security purposes.

Thus, mobile network has become one of the daily use of the human race, such as Internet, educational resource for learners, social media, advertising and business purposes. These mobile network supports voice calling and reduced the need for travel. Though the signal experience poor reception, and alteration of calls because of the high demand of number of Macro base station site from the service providers cannot be met due to the cost of operation.

A study was carried out by the Femtoforum that confirmed an increase in voice calling and data used in an indoor

This manuscript was submitted in July 21, 2016 for review, accepted on 15 October 2016. This work was supported in part by the University of Johannesburg, Johannesburg, South Africa. Department of Electrical and Electronic Engineering Sciences.

O. A. Akinlabi is a doctoral student at the Department of Electrical and Electronic Engineering Science, University of Johannesburg, Johannesburg, South Africa, SA (e-mail: akinlabiakindeji@gmail.com). environment [1]. The deployment of femtocell technology is favorable over macrocell at the indoor environment for coverage and system capacity in a low cost manner. Femtocells focuses on the quality of voice calling, data and improved signal coverage in an indoor environment for all mobile users.

In line with [1], femtocell guarantees improved coverage, capacity over the Internet backhaul with full operating capacity under the licensed spectrum at a low price for the end users. Femtocells are small base station in an indoor environment, set up by the end user and connected through the Internet, access to the mobile provide [2]-[6].

Thus femtocell supports at least four to five users at the indoor environment and is applied to residential, enterprise, hot spot and metro. The values of communication such as voice calling over networks, quality of service are linked to economic stability a country. The significance of femtocells in mobile network is that it enhance quality service and improve coverage network. Figure 1, illustrates the application of femtocell in a home setting and connections to the mobile provider through the broadband access.

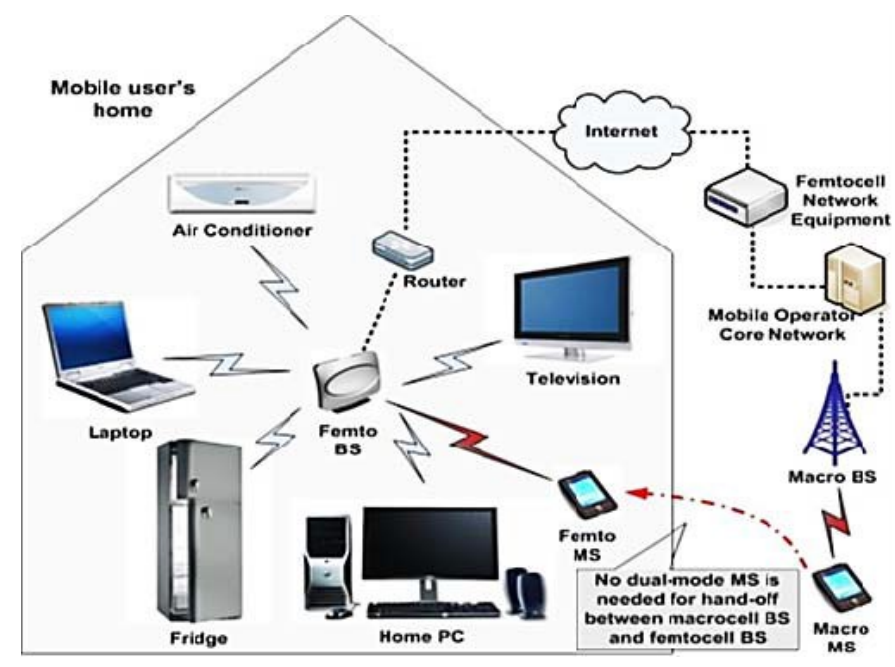

Fig. 1: Femtocell in a home setting [7]

Dr. Meera K. Joseph, is Senior Lecturer, at the School of Electrical Engineering, University of Johannesburg, Johannesburg, South Africa and she leads the ICT4D research group (e-mail: meeraj@uj.ac.za). 
The value of femtocell has contributed to the fast growth of economies and increase in mobile revenue for service providers. The market report launch by Femtoforum on femtocell has shown that it increases mobile revenue [1]. In fact, it cuts down operational cost, infrastructure and maintenance cost for mobile provide. More so, it performs certain functions of which macrocell may not perform such as it guaranteed good connectivity, home security, remote control of home appliance and quality of voice experience.

Apart of all this, it lessens the traffic load over macrocell and increase the performance of the mobile provider. Femtocell utilised the broadband for connection through the existing macrocell network for quality of voice calling, media and video streaming in which this cannot cause any problem when the provider of such broadband differs. The problem arises when they are using the same license band for the same purpose. The technical challenge is caused due to the use of the same licensed band with the existing spectrum of macrocell and ad hoc deployment of femtocell which led to interference management. But they are needed for mobile to deploy femtocell successfully for improved coverage at the indoor environment at low cost. However, the deployment of femtocells, still provides a better coverage in the network

The network traffic grew widely over the last decade due to the profitable rate flat launch by Femtoforum [1], and such request should be met by new mobile communication systems, as well as increasing the revenue. Hence, the achievement of the wireless network will depend on the providing a broadband access for mobile user, where costs per bit error rate are low [8].

Often, mobile traffic is highly demanding in homes and office environment and according to [9], more than $80 \%$ of the mobile traffic is used in an indoor environment. The new technology will offer solution for home and office, where there will be an improved reception of signals in an indoor and these promotes cost effective for the network users. The target of service provider is to satisfy the need for the high demand of mobile data in an indoor environment and also to offer an added value able service.

Mobile operator benefits mostly from these new technology known as femtocell in such a way that the operator has enough saving on coverage and profit, no more electricity bills and no time wasting on problems. Finally, it provides broadband access point in order for a connection with a satellite backhaul, for instance, inside an airplane, complex, shopping mall, train and warship respectively. These access points are modelled to be linked to the business model.

This paper is organized as follows. Related work on interference in femtocell section II. Femtocell over macrocell were explained in section III. In section IV, brief notes on the problem statement. The notation and system analysis were discussed in section V. Results based on simulations as carried out in section VI. The conclusions are drawn in VII.

\section{RELATED WORK ON INTERFERENCE IN FEMTOCELL}

The related work centered on the deployment of femtocell technology and interference as a main challenge in order to achieve the desired quality of service, good voice calling and network coverage in an indoor environment. Femtocell deployment used to achieve good connectivity to mobile users and improvement of signal behavior in an indoor environment.

The approach in [7] has practical problems due to the architecture networks constrained by number of height of radio and power emission antenna. It is more difficult to analyse the system topologies with feasible transmitter location and to find out the optimal network.

The work in [8] [9] [10] aims the optimal transmitter antenna configuration. Siomina et al. [11], offers a simulated annealing due to the central algorithm used in optimizing the channel level of the power and antenna angle. The Universal Mobile Telecommunications System (UMTS) networks in such a manner that the total channel level of the power is brought down.

As pointed out in [7], Fagen et al. carried out an algorithm that is capable to count on for the ability stage of each cell in the network in parliamentary procedure to maximize the area coverage as well as mitigate interference within a desired signal in the coverage area. In [12] the optimization of femtocell network is performed under interference in an indoor coverage using power scheme to mitigate the cause of interference among femtocell BSs is presented.

Another proposed scheme in [13] and [14], whereby a distributed utility is offered based on the SINR method at the Femtocell Access Point (FAP). FAP sets up connections to the core network over the subscriber's broadband connection and end users can enjoy improved network capacity. With the above mentioned method they provide a better output and an error estimation of SINR and intricacy of implementing the algorithm for the strength of the network.

The decentralized approach strategies that allow the Femtocell Access Point's to sense the channel and selforganize, in conditions of resource allocation and the topology of the network systems, this give a framework on game theory approach to design decentralized mechanisms for optimization and resource allocation among each femtocell user. Recently, game theory has been employed as a powerful too, which is used for the systematic analyses of the resource allocation strategies among the radio nodes [11] have been proposed for cognitive radio.

The work presented in [20] focus on the signal strength of the deployment of femtocell over macrocell where they are poor reception of signals for indoor users. It proved that the signal of the femtocell is improved over macrocell but the limitation of the study is that it does not emphasis on the transmitting 
power to avoid interference that reduces the functioning capacity of femtocell technology.

\section{FEMTOCELL OVER MACROCELL}

Thus, femtocell technology aimed is to cognitive abilities for the purpose of mobile communication, traffic loading, capacity, and coverage optimization over others mobile cellular network at the indoor [2]. Wi-Fi network is generally applicable mostly to all cellular service providers due to the strength of the signal, but the femtocell technology is much better off in terms of improved signal strength, security purpose, and voice calling service at home or office environment. However, the received signal strength gets improved due to the functionality of femtocell technology as a base station in an indoor environment. A macrocell transmit in a wide range with high transmission power that cover up to about 20miles radius due to a base station.

\begin{tabular}{|l|l|l|l|}
\hline \multirow{2}{*}{ NO. } & \multicolumn{3}{|c|}{ Table 1: Femtocell and other cellular networks } \\
\cline { 2 - 4 } & $\begin{array}{c}\text { Table column } \\
\text { subhead }\end{array}$ & \multicolumn{1}{|c|}{ Femtocell } & \multicolumn{1}{c|}{ Macrocell } \\
\hline 1 & Data Rate & $45 \mathrm{Mbps}$ & Non \\
\hline 2 & Installation & Customer & Operator \\
\hline 3 & Rent of Site & No Site Rentage & Rentage of Site \\
\hline 4 & $\begin{array}{l}\text { Operating } \\
\text { Frequency }\end{array}$ & $2,6 \mathrm{GHz}$ & $5 \mathrm{GHz}$ \\
\hline 5 & Power Ranges & $10 \mathrm{dBm}$ & $25 \mathrm{dBm}$ \\
\hline 5 & Primary Service & $\begin{array}{l}\text { Quality of voice } \\
\text { calling, Multi-media, } \\
\text { Video and security }\end{array}$ & $\begin{array}{l}\text { Data and voice } \\
\text { calling }\end{array}$ \\
\hline
\end{tabular}

In table 1, we provide the dissimilarity between both technologies used for communication transmission [13]. In order to mitigate interference femtocell must transmit at a lower power. What really distinguished femtocell technology is the valued added service introduced by the mobile provider for end users at the home or office environment. It is a service that a mobile provider is always enthusiastic to integrate as much as possible.

\section{PROBLEM STATEMENT}

Femtocell technology has immensely improved signal strength of the mobile network, but there are challenges that need to be controlled by the mobile provider due to the fact that it shares the same spectrum with the existing network. There are two cases of interference in two tier architecture networks such as co-tier and cross-tier interference. Mostly this is caused by unwanted transmitting signal within the frequency band, which gives rise to interference. Hence, the absence of interference mitigation will interfere in the quality of femtocell deployment within the network. Other challenges that faced deployment of femtocell were mobility movement and handover, self-organization, access mode and synchronization and timing etc. Our focus is on interference which is caused by the high transmitting power of Femtocell Access Point of the users. This should be the main concern for the mobile operators.

\section{NOTATION AND SYSTEM ANALYSIS}

Here we present the system notation, and parameter for network performance results. Our primary concern is to achieve an improved signal and quality of service. The estimation of SINR [14] is highly important, which is expressed as (1):

$$
\operatorname{Sin} r=\frac{g_{f b s} p_{f b s}}{\sigma+\sum p_{f b s}+\sum p_{m b s}}
$$

$p_{f b s} \ldots .$. Transmitting power of the femto base station

$p_{m b s} \ldots . .$. Transmitting power of the macrocell base station

$g_{f b s} \ldots .$. Channel gain

$\sigma$....... Noise.

In this paper, we used the path loss model [15]. These path loss models are approximations of the instability of signal behavior in an indoor environment. Therefore, the path loss is given [15] in the equation (2):

$$
\begin{aligned}
& P L(d B)=\max \left(15.3+37.6 \log _{10}(d)\right), 38.4 \\
& +20 \log _{10}(d)+0.7 d_{2 D, \text { indoor }} \\
& +18.3 n^{\left(\frac{n+2}{n+1}\right)-0.46}+q L_{i w}+L_{o w 1}+q L_{o w 2}
\end{aligned}
$$

Where, $P L$ is the path loss model

$n$---- Number of penetration floors

$q$---- Number of walls in the flats

$L_{i w}$------ Penetration Loss of the wall that different the apartment

$0.7 d_{2 D \text {,indoor }}$------ Penetration Loss by the walls inside the flats

$d$----- Distance between transmitter and receiver in meter

$L_{\text {ow }}$--------- Penetration Loss of outdoor wall

$L_{\mathrm{ow}}$ and $L_{\mathrm{iw}}$ are set to $20 \mathrm{~dB}$ and $5 \mathrm{~dB}$ respectively.

Then, we presumed that the capacity saved as the network throughput [16], mathematically it is given as (3):

$$
T=\beta \log _{2}(1+\sin r)
$$

Where $\mathrm{T}$ is the throughput and sinr is the signal. Here, a number of femtocells are selected to be used in the indoor environment with equal service provider in this area and one outdoor macrocell. Each femtocell act well as defined in the experiment, the parameters for the system analysis are shown in Table 2 and some of the above equations as presented in [20]. 


\begin{tabular}{|l|l|l|}
\hline Nos & Table 2: Simulators Parameters \\
\hline 1 & Parameters & Values \\
\hline 2 & Scenario size & $350 \times 350$ \\
\hline 3 & Macrocell Base station & 1 \\
\hline 4 & $\begin{array}{l}\text { Femtocell as a Base } \\
\text { Station }\end{array}$ & 1 \\
\hline 5 & Bandwidth & $5 \mathrm{MHz}$ \\
\hline 6 & Noise & $-174 \mathrm{dBm} / \mathrm{Hz}$ \\
\hline 7 & Macro Tx Power & $43 \mathrm{dBm}$ \\
\hline 8 & Femto Tx Power & $10 \mathrm{dBm}$ \\
\hline
\end{tabular}

The system simulation uses MATLAB as a model of operation to analysis the signal behavior of femtocell over macrocell in an indoor environment. The real life network model takes a series of events to achieve the main objective of the goal of the application of a femtocell.

\section{RESULTS}

In this section, we observed the simulation results of deployment of femtocell for improved signal and quality of voice calling. We consider the parameters and equation in section $\mathrm{V}$. The simulation results prove the signal of both femto cells and macro cells in a cellular network for better performance in an indoor environment.

Based on the result, figure 4 illustrates the deployment of femtocell in the residential area. Here we indicate the randomness of femtocell in a network, while the colors indicate subscriber and nonsubscriber of femtocell network. Here, the FAP is randomly scattered around the area.

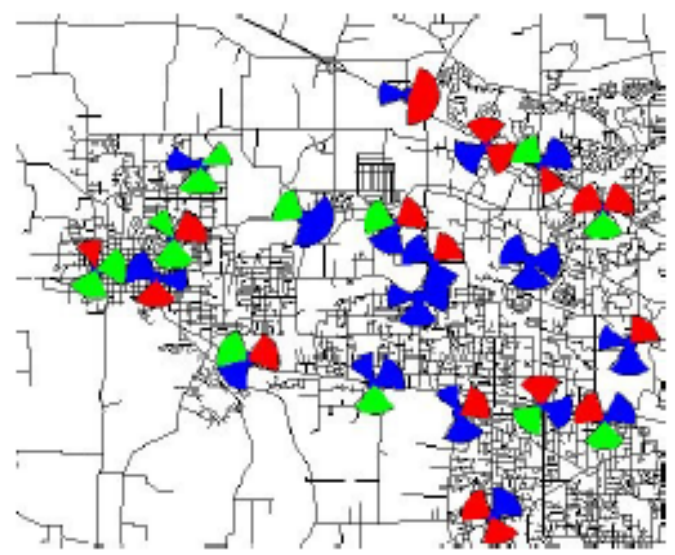

Fig 4: Deployment of femtocell network in residential area

With the simulation result shown in figure 5, bit error rate against the signal. We considered the bit error rate at the indoor environment, this is the major parameter in data transmission and communication system. From the result, we observed that the bit error rate is low and this promotes voice calling. The system throughput is obtained by a reduction of bit error rate.

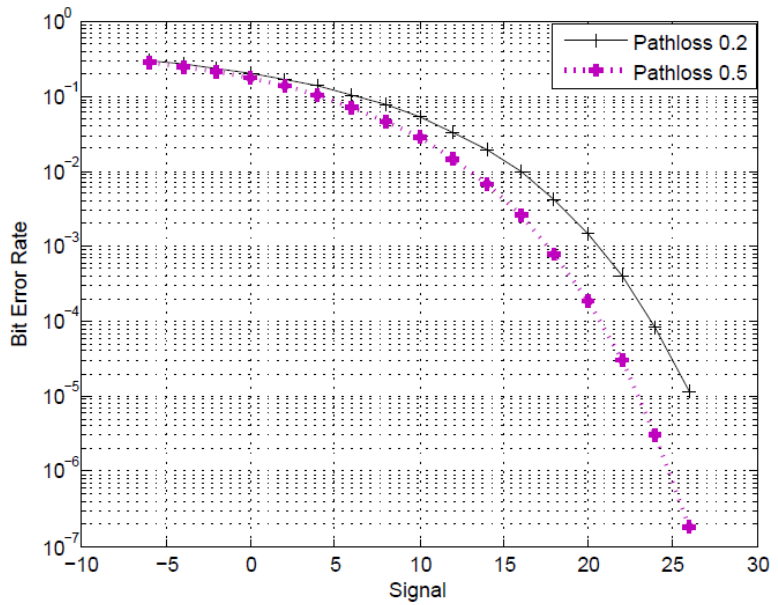

Fig. 5: Relationship between BER against the signal

In figure 6 we illustrate the throughput of both femtocell and macrocell against signal in order achieve a better signal strength at the indoor environment. With the results it was observed that the throughput of femtocell improved coverage in an indoor environment which promotes quality of service, performance for femtocell user.

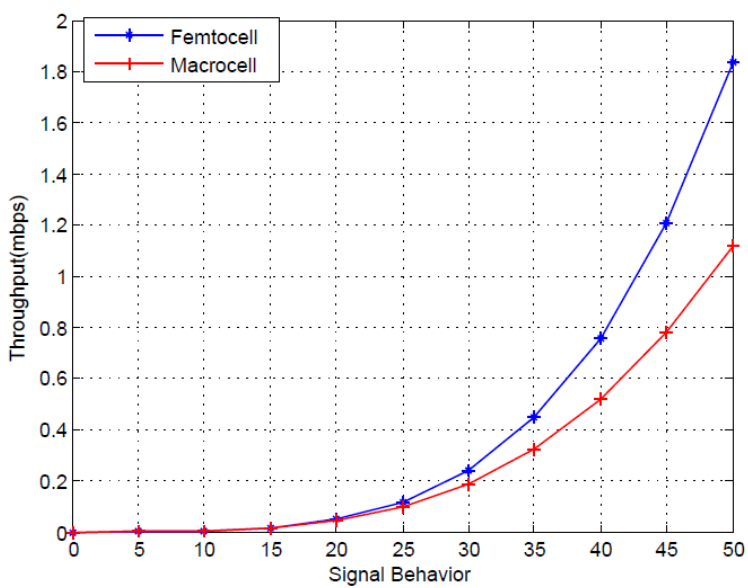

Fig 6: Signal behavior of femtocell over macrocells in an indoor environment

Although similar simulations as in Figure 5 and Figure 6 were presented in [20], in this paper we also illustrate the iteration of transmitting power against a signal behavior for both femtocell and macrocell as illustrated in Figure 7. This enables us to benchmark the transmitting power of both femtocell and macrocell in order to access an improved signal. Thus, this also shows that the power consumption is low compared to the macrocell. However, the measures for excellent performance, and quality signal, that can be used as a benchmark for femtocell deployment. 


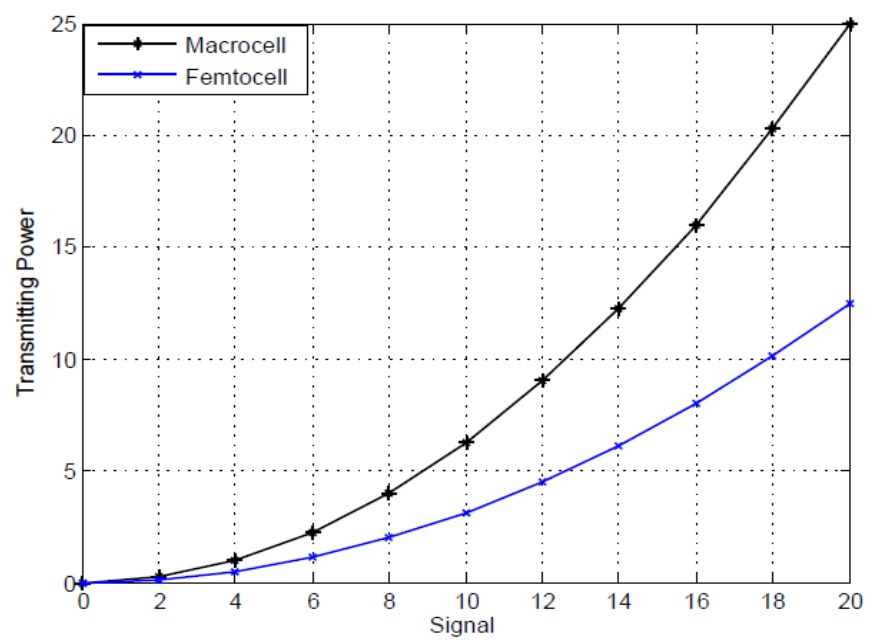

Fig 7: Iteration of transmitting power over signal behavior

\section{CONCLUSIONS}

In the past, macrocell have been the only means used for communication, data, media and many more at the indoor and outdoor environment. But due to poor reception of signals at the indoor environment femtocell was introduced. However, it has improved the reception of signals, particularly places like urban and rural environment.

It is observed that the signal performance is achieved through the deployment of femtocell technology over macrocells. The deployment of femtocells over the existing macrocell has brought efficiency and profitable solution for mobile operators. However, the cost implication of femtocell is much less than building a macrocell site, paying rent and electricity bills which is undertaken by the mobile provider. Thus, Femtocell has attracted the attention of service providers due to the valuable service and decrease of related energy consumption. Femtocells utilize the broadband connection which may be used for other applications such as video streaming. There can be associated problems when the provider of the broadband service differs from the mobile network provider. There will be cost drawbacks considering the installation in certain residential areas.

The performance capacity is attained since of the femtocell served as the Base Station (BSs) to the users in an indoor environment. The deployment of femtocell will continue to play a vital role in the market for mobile operator's network. Further work will be that of cost function implementation over power transmitting for all Femto users in the cellular network. This will allow to reduce interference within the users since the transmitting power is bench on $10-15 \mathrm{~dB}$ for better signal used.

\section{REFERENCES}

[1] Femtocell forum http:// www.femtoforum.org.com Accessed 20 October 2015

[2] 3GPP work items on Self-Organizing Networks. (Accessed 15 August 2015). [Online]. Available: http://www.3gpp.org/ftp/Information

[3] ] D. L. Perez, G. D. la Roche, A. Valcarce, A. Juttner, and J. Zhang, "Interference avoidance and dynamic frequency planning for WiMAX femtocells networks," 11th IEEE Singapore International Conference on Communication Systems, pp. 1579-1584, 19-21 Nov. 2008.
[4] M. Yavuz, F. Meshkati, S. Nanda, A. Pokhariyal, N. Johnson, B. Roghothaman, and A. Richardson, "Interference management and performance analysis of umts/hspa+ femtocells," IEEE Commun. Mag., vol. 47, no. 9, pp. 102-109, Sep. 2009.

[5] H. Claussen, "Performance of macro and co channel femtocells in a hierarchical cell structure," IEEE 18th International Symposium on Personal, Indoor and Mobile Radio Communications, pp. 1-5, 3-7 Sep. 2007.

[6] R. Baines, "The need for WiMAX Pico cell and femtocells," WiMax London, pp. 1-36, 25-26 April 2007.

[7] Femtocell Base Station compared to Macrocell Base Station:Available online $\quad$ http://www.analog.com/library/analogdialogue/archives/4212/AD42 12 FIG-01.jpg

[8] T. Giles, J. Markendahl, J. Zander, P. Zetterberg, P. Karlsson, G. Malmgren, J. Nilsson, “ Cost drivers and deployment scenarios for future broadband wireless networks- key research problems and directions for research" IEEE 59 ${ }^{\text {th }}$ Vehicular Technol. Conference, VTC 2004- Spring 4, 2042-2046 (2004) 17-19 May 2004, Milan (Italy).

[9] Fagen, P.A. Vicharelli, J. Weitzen, "AutomatedWireless Coverage Optimization With Controlled Overlap," IEEE Transactions on VehicularTechnology, vol. 57, no. 4, pp. 2395-2403, Jul 2008.

[10] Y. Sun, F. Gunnarsson, K. Hiltunen, "CPICH Power Settings in Irregular WCDMA Macro Cellular Networks," in Proc. PIMRC, Beijing, China, Sep 2003, pp. 1176-1180.

[11] M. Garcia-Lozano, S. Ruiz, J.J. Olmos, "UMTS Optimum Cell Load Balancing for Inhomogeneous Traffic Patterns," in Proc. IEEE VTC, Los Angeles, CA, USA, Sep 2004, pp. 909-913.

[12] K. Valkealahti, A. H"oglund, J. Parkkinen, A. Hamalainen, "WCDMA Common Pilot Power Control for Load and Coverage Balancing," in Proc. PIMRC, Lisbon, Portugal, Sep 2002, pp. 1412-1416.

[13] I. Siomina, P. V"arbrand, D. Yuan, "Automated Optimization of Service Coverage and Base Station Antenna Configuration in UMTS Networks," IEEE Wireless Communications, vol. 13, no. 6, pp. 16-25, Dec 2006.

[14] Kwanghun Han, Youngkyu Choi, Dongmyoung Kim, Minsoo Na, Sunghyun Choi, Kiyoung Han, "Optimization of femtocell network configuration under interference constraints," 7th International Symposium on Modelling and Optimization in Mobile, Ad Hoc, and Wireless Networks, pp. 1-7, 23-27 Jun. 2009.

[15] V. Chandrasekhar, J.G. Andrews, T. Muharemovic, Zukang Shen, A. Gatherer, "Power control n two-tier femtocell networks," , IEEE Transactions on Wireless Communications, vol. 8, no. 8, pp. 4316-4328, Aug. 2009.

[16] V. Chandrasekhar, J.G. Andrews, S. Zukang, T. Muharemovic, A. Gatherer, "Distributed Power Control in Femtocell-Underlay Cellular Networks," IEEE Global Telecommunications Conference, pp.1-6, Nov. 30-Dec. 42009.

[17] D Lo'pez-Pe'rez, A Valcarce, A' kos Lada'nyi, G de la Roche, and J Zhang, "Femtocell versus Wi-Fi: A Survey and Comparison of Architecture and Performance", IEEE Wireless Communications, Vol. pp no. 916-920, 2009. 13

[18] P. Lee, T. Lee, J. Jeong, and J. Shin, "Interference Management in Lte Femtocell Systems Using Fractional Frequency Reuse," in Advanced Communication Technology (ICACT), 2010 The $12^{\text {th }}$ International Conference on, vol. 2, Feb. 2010, pp. $1047-1051$.

[19] B. S. L. Castro, I. R. Gomes, F. C. J. Ribeiro, G. P. S. Cavalcarite, "COST231-Hatta and SUI Models Performance using a LMS Tuning Algorithm on $5.86 \mathrm{~Hz}$ in Amazon Region Cities" Proc. Of IEEE. EUCAP2010. ISBN: 978-847653-472-4, pp. 1-3, Jul. 2010.

[20] O.A. Akinlabi, M.K. Joseph "Signal behaviour in an indoor environment; Femtocell over Macrocell" $16^{\text {th }}$ IEEE International Conference on Environment and Electrical Engineering, pp, 7-10 June 2016. Florence, Italy. 


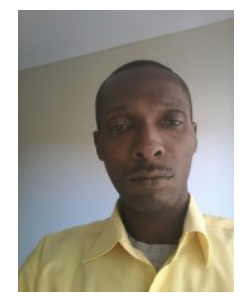

Akinlabi Olaniyi Akindeji was born in Surulere, Lagos State, Nigeria, and West Africa country in 1978 $\mathrm{He}$ received the B.Tech in Electrical and Electronic Engineering from Rivers State Polytechnic, Rivers state, Nigeria and his M.Tech. Degree in Electrical Engineering from the University of Johannesburg (UJ) in 2014. The author become a member of SAIEE in 2013. He is currently enrolled for DPhil Electrical and Electronic Engineering at UJ. For academic excellence, he has the best paper award from IMECS 2014. He has many research papers to his credit already. His research interest focuses on Information and Communications Technology and power distribution and generation and femtocells.

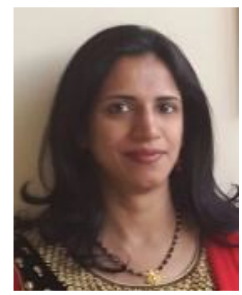

Meera K. Joseph received the degrees of DPhil. Engineering Management from the University of Johannesburg (UJ) in 2013, and M.C.A in 1997 from the Bangalore University. She works as a Senior Lecturer at UJ and is a Professional Member of IITPSA. Many post graduate students completed under her supervision and she has many IEEE international conference papers, Journal papers and book chapters to her credit. She runs the ICT4D research group in the School of Electrical, UJ. Her research interests include Information and Communication Technology for Development (ICT4D), smart grids, Femtocells, cloud computing and wireless networks. 